\title{
Control of HOD photodissociation dynamics via bond-selective infrared multiphoton excitation and a femtosecond ultraviolet laser pulse
}

\section{Amstrup, Bjarne; Henriksen, Niels Engholm}

Published in:

Journal of Chemical Physics

Link to article, DOI:

$10.1063 / 1.463399$

Publication date:

1992

Document Version

Publisher's PDF, also known as Version of record

Link back to DTU Orbit

Citation (APA):

Amstrup, B., \& Henriksen, N. E. (1992). Control of HOD photodissociation dynamics via bond-selective infrared multiphoton excitation and a femtosecond ultraviolet laser pulse. Journal of Chemical Physics, 97(11), 82858295. https://doi.org/10.1063/1.463399

\section{General rights}

Copyright and moral rights for the publications made accessible in the public portal are retained by the authors and/or other copyright owners and it is a condition of accessing publications that users recognise and abide by the legal requirements associated with these rights.

- Users may download and print one copy of any publication from the public portal for the purpose of private study or research.

- You may not further distribute the material or use it for any profit-making activity or commercial gain

- You may freely distribute the URL identifying the publication in the public portal 


\title{
Control of HOD photodissociation dynamics via bond-selective infrared multiphoton excitation and a femtosecond ultraviolet laser pulse
}

\author{
Bjarne Amstrup and Niels E. Henriksen \\ Chemistry Department B, The Technical University of Denmark, DTH 301, DK-2800 Lyngby, Denmark
}

(Received 20 April 1992; accepted 24 August 1992)

\begin{abstract}
A scheme for controlling the outcome of a photodissociation process is studied. It involves two lasers-one intense laser in the infrared region which is supposed to excite a particular bond in the electronic ground state, and a second short laser pulse in the ultraviolet region which, at the right moment, excites the molecule to a dissociative electronic state. We consider the HOD molecule which is ideal due to the local mode structure of the vibrational states. It is shown that selective and localized bond stretching can be created in simple laser fields. When such a nonstationary vibrating HOD molecule is photodissociated with a short laser pulse ( $\sim 5 \mathrm{fs}$ ) complete selectivity between the channels $\mathrm{H}+\mathrm{OD}$ and $\mathrm{D}+\mathrm{OH}$ is observed over the entire absorption band covering these channels.
\end{abstract}

\section{INTRODUCTION}

Bond-selective excitations and its use in controlling the outcome of chemical reactions is a subject of much current interest. ${ }^{1}$ A nice example is the photodissociation of HOD in the first electronically excited state. The vibrational eigenstates of the electronic ground state are essentially local modes in the $\mathrm{OH}$ or OD stretch. The states are either pure $\mathrm{OH}$ or $\mathrm{OD}$ excitations or combinations thereof. If, e.g., an excited $\mathrm{OH}$ eigenstate is populated in HOD (we can denote such a molecule as $\mathrm{H}-\mathrm{OD}$ ), an enhanced branching into the channel $\mathrm{H}+\mathrm{OD}$ is observed in a subsequent photodissociation process. ${ }^{2-6}$ This has been demonstrated theoretically as well as experimentally. The setup involved two lasers in the $\mathrm{cw}$ regime (i.e., pulsed lasers with a long pulse duration were used in the experiments) the first laser prepares the vibrational eigenstate and the second laser dissociates this state via an excitation to the first electronically excited state. The branching ratio between $\mathrm{H}+\mathrm{OD}$ and $\mathrm{D}+\mathrm{OH}$ was analyzed as a function of the frequency of the second laser. It was shown that this ratio depends on the frequency. High selectivity could be obtained, however, only in a narrow frequency region. This frequency region is broadened when the initial vibrational excitation is increased but is still relative narrow even at high vibrational excitation.

The question we are considering in this paper is: Can we control the branching ratio in HOD photodissociation completely, i.e., at all frequencies obtain the desired product-and what will it take to do so? The scheme we are suggesting here is a modified form of the scheme mentioned above. In order to obtain complete selectivity appropriate linear combinations of the local mode eigenstates in HOD must be created. We study therefore first the possibility of creating selective and localized bond stretching via simple intense infrared (ir) laser fields. The HOD molecule seems to be an ideal candidate where, in addition, a good potential energy surface exists. Using intense ir laser fields, we will consider bond-selective multiphoton transi- tions and, in particular, the possibility of creating localized wave packets in $\mathrm{OH} / \mathrm{OD}$, respectively, corresponding as far as possible to a vibrating bond in a classical sense.

In connection with controlling the outcome of chemical reactions, a vibrating (nonstationary) molecule impose restrictions on the optimal timing concerning its subsequent use in chemical reactions (e.g., in photodissociation). The outcome of subsequent reactions can depend on the bond length as well as the momentum in the excited bond. This active control scheme should be contrasted with passive control via bond-selected vibrational eigenstates which is easier to handle, but, on the other hand, it does not hold the same potential as active control due to the delocalized structure of vibrational eigenstates. Thus the present study looks into a (conceptually) simple way to extend the control in HOD photodissociation and similar processes.

The present study is obviously also related to previous work on multiphoton excitation of molecules as well as other control schemes. Like the studies by Manz and coworkers on multiphoton excitation of water $^{7,8}$ (see also Ref. 9) and the pump-dump scheme suggested by Tannor and Ricc. ${ }^{10,11}$ The scope and aim of these studies werc, however, somewhat different from the present study. Another interesting active control scheme for unimolecular reactions has been introduced by Brumer and Shapiro. ${ }^{12,13}$

This paper is organized in the following way: Section II defines the Hamiltonian of HOD in a laser field and describes the numerical technique used in order to solve the time-dependent Schrödinger equation. The vibrational dynamics of the electronic ground state induced by ir multiphoton excitation is presented and discussed in Sec. III. In Sec. IV the nuclear dynamics in the first (dissociative) electronically excited state is analyzed for photodissociation out of various initial vibrational states. The results are summarized in Sec. $\mathrm{V}$. 


\section{THEORY AND MODEL}

We consider the two degree of freedom model of HOD where bending and rotation is neglected. ${ }^{2}$ The coordinates describing the $\mathrm{O}-\mathrm{H}$ and $\mathrm{O}-\mathrm{D}$ bonds are denoted by $r_{1}$ and $r_{2}$, respectively. The associated conjugate momenta are $p_{1}$ and $p_{2}$. The internal kinetic energy operator is given by

$$
\widehat{T}=\frac{\hat{p}_{1}^{2}}{2 \mu_{1}}+\frac{\hat{p}_{2}^{2}}{2 \mu_{2}}+\frac{\hat{p}_{1} \hat{p}_{2}}{m_{\mathrm{O}}} \cos \theta,
$$

where

$$
\begin{aligned}
& \hat{p_{j}}=\frac{\hbar}{i} \frac{\partial}{\partial r_{j}}, \quad j=1,2, \\
& \mu_{1}=m_{\mathrm{H}} m_{\mathrm{O}} /\left(m_{\mathrm{H}}+m_{\mathrm{O}}\right), \\
& \mu_{2}=m_{\mathrm{O}} m_{\mathrm{D}} /\left(m_{\mathrm{O}}+m_{\mathrm{D}}\right),
\end{aligned}
$$

and $\theta$ is the fixed bending angle of $104.52 \mathrm{deg}$.

Two electronic states of the molecule are considered: the electronic ground state and the first electronically excited state. An intense laser in the infrared (ir) region and a (weak) laser in the ultraviolet (uv) region of the spectrum are interacting with the molecule. The intensity of the second laser is so low that stimulated emission from the excited electronic state can be neglected. The time evolution associated with the nuclear motion can then be calculated from the time-dependent Schrödinger equation,

$$
i \hbar \frac{\partial}{\partial t}\left(\begin{array}{c}
\psi_{g} \\
\psi_{e}
\end{array}\right)=\left(\begin{array}{cc}
\hat{H}_{g}+\hat{H}_{\mathrm{ir}}(t) & \hat{H}_{\mathrm{uv}}(t) \\
\hat{H}_{\mathrm{uv}}(t) & \hat{H}_{e}
\end{array}\right)\left(\begin{array}{l}
\psi_{g} \\
\psi_{e}
\end{array}\right),
$$

where $\psi_{g} \equiv \psi_{g}\left(r_{1}, r_{2}, t\right)$ and $\psi_{e} \equiv \psi_{e}\left(r_{1}, r_{2}, t\right)$ are the wave functions associated with nuclear motion in the ground and first excited electronic state, respectively. $\hat{H}_{g}=\widehat{T}+V_{g}$ and $\widehat{H}_{e}=\widehat{T}+V_{e}$ are the nuclear Hamiltonians in the two states. $\hat{H}_{\mathrm{ir}}(t)$ and $\hat{H}_{\mathrm{uv}}(t)$, to be specified below, describe the interaction between the molecule and the two lasers.

Equation (3) is solved numerically on two twodimensional grids, with the initial condition $(t=0)$ that $\psi_{g}$ is the vibrational ground state of HOD,

$$
\psi_{g}\left(r_{1}, r_{2}, t=0\right)=\left\langle r_{1}, r_{2} \mid n_{\mathrm{OH}}=0, n_{\mathrm{OD}}=0\right\rangle
$$

and $\psi_{e}=0$. The effect of the kinetic energy operator of the Hamiltonian is evaluated using a two-dimensional fast Fourier transform (FFT) algorithm. ${ }^{14}$ The time propagation is evaluated using the Lanczos scheme. ${ }^{15}$ The vibrational ground state is obtained via propagation in imaginary time. ${ }^{16}$

In the calculations, we have used a time step of the order of 0.5 a.u. and a grid spacing, $\delta r_{j}$, which equals 0.042 $a_{0}$. Atomic units is used throughout this article unless other units are indicated explicitly.

\section{A. Electronic ground state}

The potential energy function associated with the electronic ground state is represented as two Morse oscillators and a coupling term, ${ }^{17,18}$

$$
\begin{aligned}
V_{g}\left(r_{1}, r_{2}\right)= & D\left[1-e^{-\alpha\left(r_{1}-r_{0}\right)}\right]^{2}+D\left[1-e^{-\alpha\left(r_{2}-r_{0}\right)}\right]^{2} \\
& +f_{12}\left(r_{1}-r_{0}\right)\left(r_{2}-r_{0}\right),
\end{aligned}
$$

where $D=0.2092$ hartree, $\alpha=1.1327 a_{0}^{-1}, r_{0}=1.81 a_{0}$, and

$$
f_{12}=\frac{F_{12}}{1+e^{\beta\left[\left(r_{1}-r_{0}\right)+\left(r_{2}-r_{0}\right)\right]}},
$$

where $\beta=1.0 a_{0}^{-1}$, and $F_{12}=-6.76 \times 10^{-3}$ hartree $/ a_{0}^{2}$.

The interaction with the laser field is described semiclassically in the electric dipole approximation. Thus

$$
\hat{H}_{\mathrm{ir}}(t)=-\boldsymbol{\mu}_{g}\left(\mathbf{r}_{1}, \mathbf{r}_{2}\right) \cdot \mathbf{E}_{0} a(t) \cos \omega_{\mathrm{ir}} t,
$$

where $a(t)$ describes the shape of the pulse. We consider a rectangular pulse, i.e.,

$$
a(t)=\left\{\begin{array}{cc}
0 & t<0 \\
1 & 0 \leqslant t \leqslant t_{l} \\
0 & t>t_{l}
\end{array}\right.
$$

where $t_{l}$ gives the pulse length and a Gaussian pulse,

$$
a(t)=\left(\frac{8 \gamma t_{l}^{2}}{\pi}\right)^{1 / 4} \exp \left[-\gamma\left(t-t_{\mathrm{ir}}\right)^{2}\right]
$$

which is normalized such that it carries the same energy as the rectangular pulse of length $t_{l}$. The pulse length of the Gaussian pulse, defined as the full width at half-maximum (FWHM), is $\sqrt{4 \ln 2 / \gamma}$.

The electric dipole moment function of the electronic ground state is described by a bond dipole model, ${ }^{19}$

$\mu_{g}\left(\mathbf{r}_{1}, \mathbf{r}_{2}\right)=\mathbf{r}_{1} \mu_{0} \exp \left(-r_{1} / r^{*}\right)+\mathbf{r}_{2} \mu_{0} \exp \left(-r_{2} / r^{*}\right)$,

where $\mu_{0}=7.85 \mathrm{D} / \AA \hat{\mathrm{A}}$ and $r^{*}=0.6 \AA$. The HOD molecule is placed in the $x z$ plane with the $z$ axis as bisector. With an $x$-polarized electric field of the exciting laser, the interaction takes the form,

$$
\hat{H}_{\mathrm{ir}}(t)=\left[\mu_{1}\left(r_{1}\right)-\mu_{2}\left(r_{2}\right)\right] A_{0} a(t) \cos \omega_{\mathrm{ir}} t,
$$

where

$$
\begin{aligned}
& \mu_{j}\left(r_{j}\right)=\mu_{0} r_{j} \exp \left(-r_{j} / r^{*}\right), \quad j=1,2, \\
& A_{0}=E_{0} \cos \phi,
\end{aligned}
$$

and $\phi=(\pi-\theta) / 2$.

Little is known about the exact form of the dipole moment. $A b$ initio calculations on the dipole moment of water seems only to be available in a small region around the equilibrium configuration. ${ }^{20}$ This region is, however, too narrow for the present conditions where the bonds can execute large amplitude motion. When we compare the first derivative with respect to a bond distance (leaving the other bond in its equilibrium position) at the equilibrium position the agreement between the model function and the $a b$ initio calculation is poor. Thus the derivative of the model function is $-0.949 \mathrm{D} / \AA$, whereas the $a b$ initio cal- 
culation gives $0.395 \mathrm{D} / \AA$. Whether this disagreement is due to inaccurate $a b$ initio data or a deficiency in the model function is not clear. The model function was, however, fitted to some experimental data. ${ }^{19}$ Anyhow, it is expected to reproduce nothing but the qualitative behavior of the dipole moment.

\section{B. Photodissociation in the first excited electronic state}

The potential energy function of the first electronically excited state, $V_{e}$, is known from $a b$ initio calculations. ${ }^{21,22}$ This $a b$ initio surface is used in our calculations. The transition dipole function was also calculated by $a b$ initio methods but only in a relatively small region $\left(r_{1}, r_{2} \leqslant 2.6 a_{0}\right)$ which is too small for the present purpose. Therefore, we use the expression, ${ }^{3}$

$$
\mu_{g e}=\frac{2.225}{1+e^{\beta\left(r_{1}-r_{0}\right)}} \times \frac{2.225}{1+e^{\beta\left(r_{2}-r_{0}\right)}},
$$

which is constructed such that it gives values similar to the $a b$ initio data and, at the same time, goes smoothly to zero as the bonds dissociate.

The transition dipole moment vector, $\boldsymbol{\mu}_{g e}$, is perpendicular to the molecular plane. ${ }^{22}$ We assume that the electric field vector of the uv-laser field is parallel to this vector and the interaction with the laser takes the form,

$$
\hat{H}_{\mathrm{uv}}(t)=-\mu_{g e}\left(r_{1}, r_{2}\right) E_{0} a(t) \cos \omega_{\mathrm{uv}} t .
$$

We consider an ultrashort ( $\delta$ function) pulse,

$$
a(t)=\delta\left(t-t_{\mathrm{uv}}\right)
$$

and a Gaussian pulse shape,

$$
a(t)=\exp \left[-\gamma\left(t-t_{\mathrm{uv}}\right)^{2}\right]
$$

\section{DYNAMICS IN THE ELECTRONIC GROUND STATE}

We consider here vibrational dynamics induced by the intense ir laser. In order to find appropriate resonance conditions, the vibrational energies are needed. The energy and assignment of the first 16 vibrational eigenstates of HOD as found by integrating the Schrödinger equation in imaginary time ${ }^{16}$ is given in Table $\mathrm{I}$. Compared to available experimental data, ${ }^{17}$ the deviation is less than $0.25 \%$. The last two columns gives a comparison to the energy and wave functions of the corresponding states in the two uncoupled Morse oscillators. The effect of the coupling terms in the kinetic energy and the potential for HOD is, accordingly, very small for the states considered here. The effect on the energy spacing is negligible. When contour plots of the higher vibrational states are inspected the effect of the coupling is seen as a small rotation of the eigenstates, they are not completely parallel to the $\mathrm{OH}$ and $\mathrm{OD}$ axis, respectively. The vibrational ground state $|0,0\rangle$ superimposed on the associated potential energy surface (dashed lines) is shown in Fig. 1. This is the initial state $(t=0)$ for the vibrational dynamics considered in the following.

We consider first a laser pulse with a rectangular pulse shape as defined by Eq. (8). The effective intensity
TABLE I. Energy and assignment of the first 16 vibrational eigenstates in HOD. The energy is measured relative to the vibrational ground state. $n_{\mathrm{OH}}$ and $n_{\mathrm{OD}}$ denotes the number of vibrational quanta in $\mathrm{OH}$ and $O D$, respectively. $\Delta E$ is the energy of the two corresponding uncoupled Morse oscillator states, minus $E_{n_{\mathrm{OH}}, n_{\mathrm{OD}}}$. The last column gives the overlap between the eigenstate and the product of the two corresponding states in the uncoupled Morse oscillators, i.e., $S_{n_{\mathrm{OH}}, n_{\mathrm{OD}}}$ $=\mid\left\langle n_{\mathrm{OH}}\left|\left\langle n_{\mathrm{OD}} \mid n_{\mathrm{OH}}, n_{\mathrm{OD}}\right\rangle\right|^{2}\right.$.

\begin{tabular}{ccccccc}
\hline \hline$E_{n_{\mathrm{OH}}, n_{\mathrm{OD}}}\left(\mathrm{cm}^{-1}\right)$ & $n_{\mathrm{OH}}$ & $n_{\mathrm{OD}}$ & $\left\langle r_{\mathrm{OH}}\right\rangle$ & $\left\langle r_{\mathrm{OD}}\right\rangle$ & $\Delta E\left(\mathrm{~cm}^{-1}\right)$ & $S_{n_{\mathrm{OH}}, n_{\mathrm{OD}}}$ \\
\hline 0 & 0 & 0 & 1.84 & 1.83 & 0 & 1.000 \\
2728 & 0 & 1 & 1.84 & 1.87 & -2 & 0.999 \\
3706 & 1 & 0 & 1.90 & 1.83 & 1 & 0.999 \\
5369 & 0 & 2 & 1.84 & 1.92 & -4 & 0.997 \\
6433 & 1 & 1 & 1.90 & 1.87 & -2 & 0.994 \\
7250 & 2 & 0 & 1.96 & 1.83 & 3 & 0.997 \\
7925 & 0 & 3 & 1.84 & 1.96 & -5 & 0.997 \\
9073 & 1 & 2 & 1.90 & 1.92 & -5 & 0.990 \\
9975 & 2 & 1 & 1.96 & 1.87 & -2 & 0.987 \\
10395 & 0 & 4 & 1.84 & 2.01 & -6 & 0.996 \\
10632 & 3 & 0 & 2.02 & 1.83 & 5 & 0.993 \\
11628 & 1 & 3 & 1.90 & 1.96 & -7 & 0.988 \\
12614 & 2 & 2 & 1.96 & 1.92 & -6 & 0.980 \\
12778 & 0 & 5 & 1.84 & 2.06 & -7 & 0.996 \\
13354 & 3 & 1 & 2.02 & 1.87 & -2 & 0.975 \\
13852 & 4 & 0 & 2.09 & 1.83 & 9 & 0.987 \\
\hline \hline
\end{tabular}

[ $\left.\varepsilon_{0} c\left(E_{0} \cos \phi\right)^{2} / 2\right]$ is $50 \mathrm{TW} / \mathrm{cm}^{2}$ and the (central) frequency $\omega_{\mathrm{ir}}=E_{4,0} / 4=3463 \mathrm{~cm}^{-1}$. The pulse length is $t_{l}$ $=500 \mathrm{fs}=0.5 \mathrm{ps}$. Figure 2 shows $\left\langle r_{1}\right\rangle$ and $\left\langle r_{2}\right\rangle$, i.e., the expectation value of the $\mathrm{OH}$ and the $\mathrm{OD}$ bond length, respectively, and the associated uncertainties $\left(\Delta r_{i}\right.$ $\left.=\sqrt{\left\langle r_{i}^{2}\right\rangle-\left\langle r_{i}\right\rangle^{2}}, i=1,2\right)$ as a function of time. We observe a strong and highly selective excitation of the $\mathrm{OH}$ bond. Small oscillations in the OD bond are also seen. During excitation of the OH bond the population in the eigenstates $\left|n_{\mathrm{OH}}, n_{\mathrm{OD}}=0\right\rangle$ changes as a function of time. The change in the OD bond length is a reflection of the

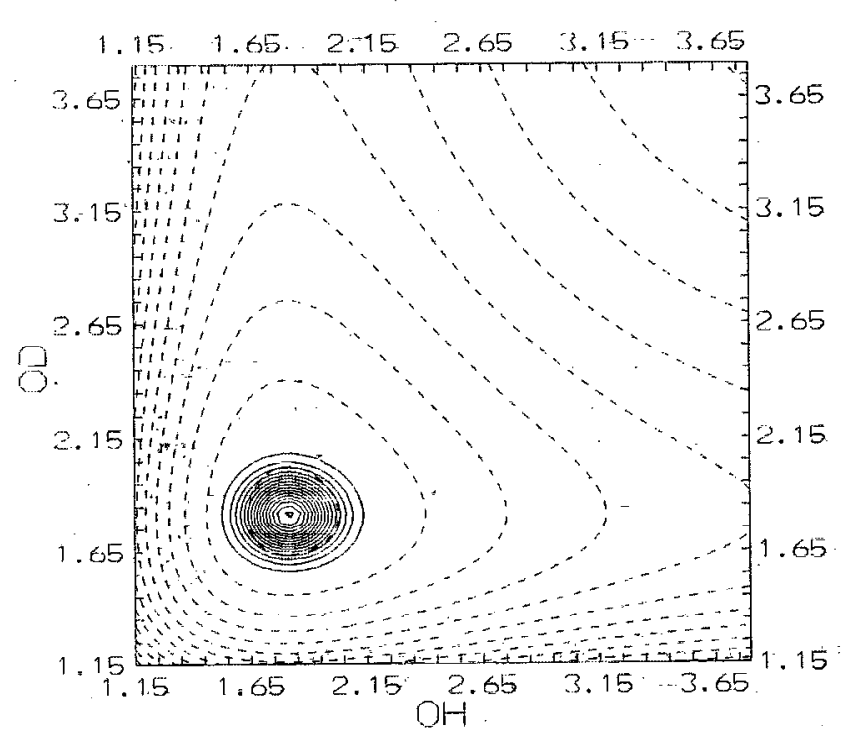

FIG. 1. Absolute square of the wave function for the vibrational ground state $|0,0\rangle$ on the potential energy surface of the electronic ground state of HOD (dashed lines). 

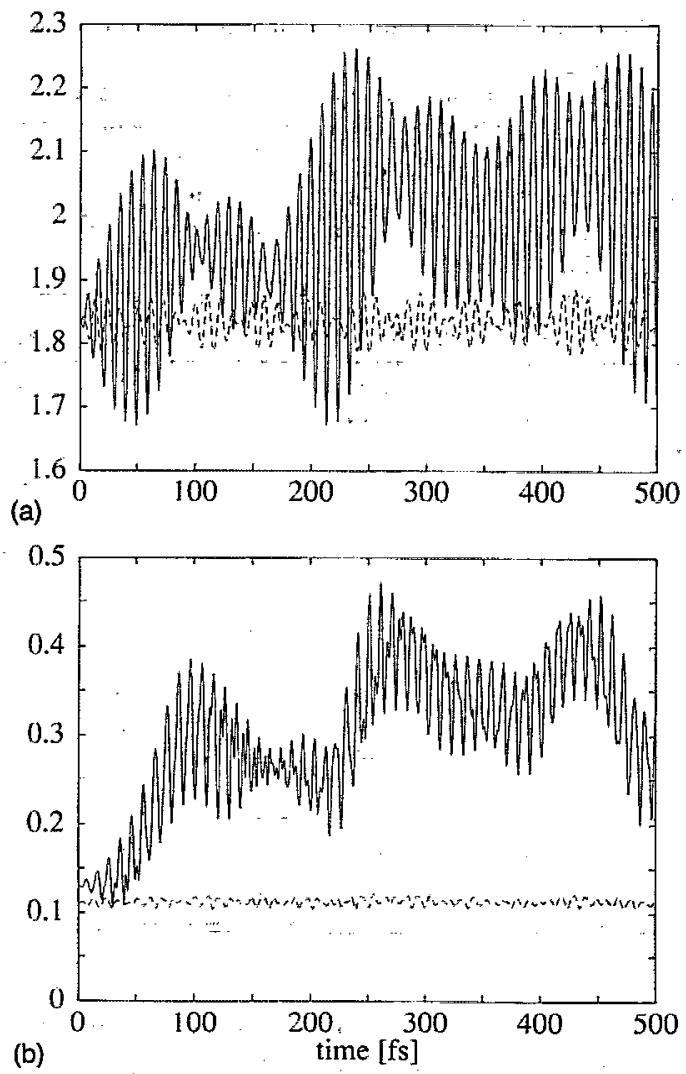

FIG. 2. (a) Average bond lengths and (b) associated uncertainties (OH full line, OD dashed line) as a function of time (femtoseconds) under multiphoton excitation using a laser with rectangular pulse shape. The frequency is $\omega_{\mathrm{ir}}=3463 \mathrm{~cm}^{-1}$.

small coupling between the two degrees of freedom which affects the eigenstates increasingly as the excitation in the $\mathrm{OH}$ bond is increased. The $\mathrm{OH}$ bond oscillates for short times ( $t<40 \mathrm{fs}$ ) much like a (linearly) forced harmonic oscillator whereas the dynamics for longer times gets more and more influenced by the anharmonicity of the potential, which implies that the coherence in the wave packet is lost. This behavior has been observed before ${ }^{23}$ for the dynamics of a Morse oscillator under multiphoton excitation. In Fig. 3 the frequency is changed to $\omega_{\mathrm{ir}}=E_{0,4} / 4=2599 \mathrm{~cm}^{-1}$. Now the OD stretch is selectively excited.

A Gaussian pulse ( $F W H M=50 \mathrm{fs}, t_{\mathrm{ir}}=100 \mathrm{fs}$ ) as defined by Eq. (9) is considered next. Figure 4 shows the expectation value of the $\mathrm{OH}$ and $\mathrm{OD}$ bond length, respectively, and the associated uncertainties as a function of time when $\omega_{\mathrm{ir}}=3463 \mathrm{~cm}^{-1}$. Again a strong and selectivc excitation of the $\mathrm{OH}$ bond is observed. The excitation is particularly strong at the peak of the pulse. When this result is compared to Fig. 2, it is clear that the short Gaussian pulse gives a stronger excitation than the rectangular pulse. Figure 5 shows the same picture as in Fig. 4 when $\omega_{\mathrm{ir}}=2599 \mathrm{~cm}^{-1}$, except that the roles of $\mathrm{OH}$ and $\mathrm{OD}$ are interchanged. Figures 6-8 give further details of the excitation in Fig. 5. Figure 6 shows the energy of the HOD molecule, $\left\langle\hat{H}_{g}\right\rangle$, as a function of time. The energy deposited in the OD bond is close to $50 \%$ of the dissociation
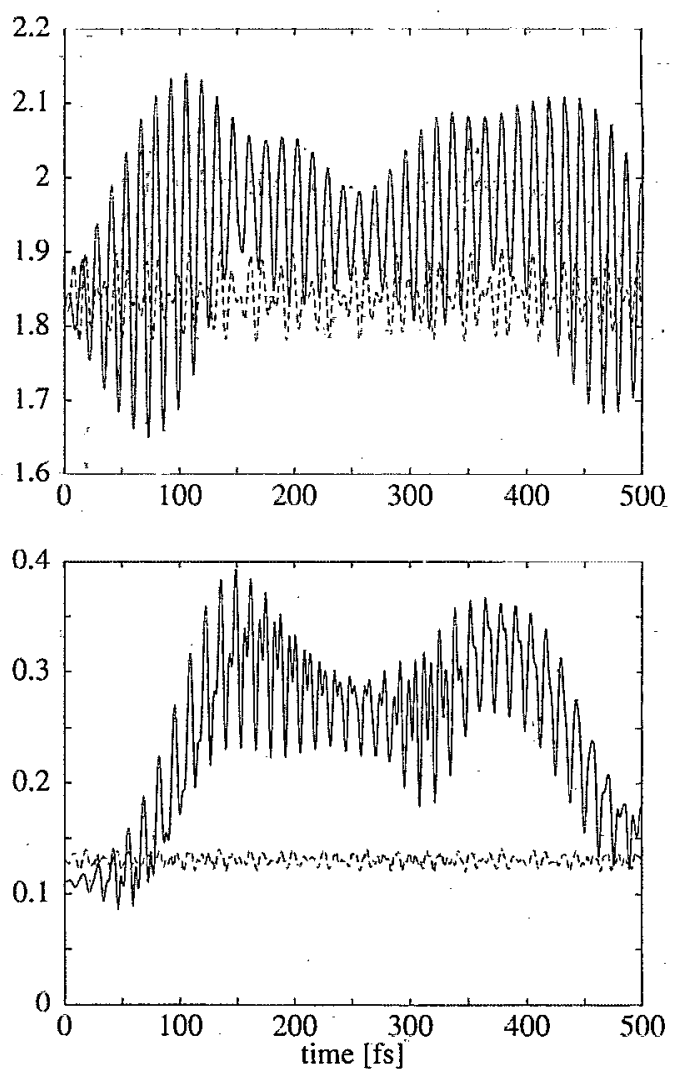

FIG. 3. Same as Fig. 2, except that $\omega_{\mathrm{ir}}=2599 \mathrm{~cm}^{-1}$, the full and dashed lines are associated with the $\mathrm{OD}$ bond and $\mathrm{OH}$ bond, respectively.

energy. The time evolution of the wave packet projections on the first 16 vibrational eigenstates (Table $I$ ) is shown in Fig. 7. Eigenstates with several vibrational quanta get a high population as a result of the high intensity of the laser. Note that only pure OD local modes are noticeably excited and that the populations are constant when the pulse has decayed. Figure 8 shows the wave packet created by the Gaussian pulse at 100,102.5, 105, and $107.5 \mathrm{fs}$. The OD bond is first compressed and stretches subsequently. At 102.5 and $105 \mathrm{fs}$ a pure OD excitation has been created which corresponds to a highly elongated OD bond with a very localized bond length distribution. At $107.5 \mathrm{fs}$ the bond is stretched even more, some of the localization is, however, lost.

The structure of the vibrational eigenstates is highly suggestive of the fact that we can selectively excite the $\mathrm{OH}$ or OD bond in HOD. In view of the very intense (and short) laser pulses considered above, the result is nevertheless not trivial. The frequency broadening due to the high intensity and the short duration could have spoiled the selectivity. In the following, the molecule is exposed to the Gaussian ir-laser pulse of Fig. 5 which selectively excites the OD bond.

\section{DYNAMICS IN THE FIRST ELECTRONICALLY EXCITED STATE}

We consider now the full setup with two lasers: An intense ir-laser as in the previous section and a (weak) uv 

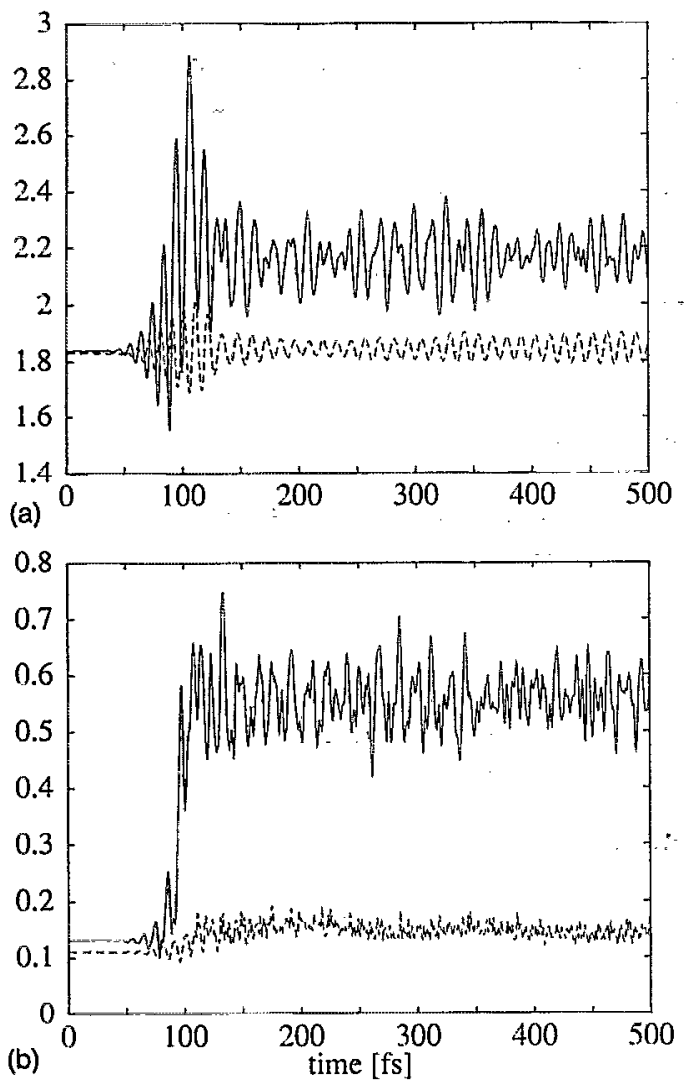

FIG. 4. (a) Average bond lengths and (b) associated uncertainties (OH full line, OD dashed line) as a function of time (femtoseconds) under multiphoton excitation using a laser with a Gaussian pulse shape. The duration of the pulse (FWHM) is $50 \mathrm{fs}$ and $\omega_{\mathrm{ir}}=3463 \mathrm{~cm}^{-1}$.

laser which can take the molecule into the excited (dissociative) electronic state. The chemistry is

$$
\mathrm{HOD} \rightarrow\left\{\begin{array}{l}
\mathrm{H}+\mathrm{OD} \\
\mathrm{D}+\mathrm{OH}^{*}
\end{array}\right.
$$

First the dissociative dynamics induced by an ultrashort $[\delta$ function, Eq. (15)] pulse is considercd.

In order to illustrate the difference between the control scheme used previously and the one suggested in the present work, we make a small digression and show in Figs. 9 and 10 the dissociation dynamics out of two vibrational eigenstates. Figure 9 shows the dissociation dynamics out of the vibrational ground state of HOD. It is clear that most of the products show up in the channel $\mathrm{H}+\mathrm{OD}$. For dissociation out of the $|0,4\rangle$ vibrational eigenstate, i.e., four vibrational quanta in the OD bond, the picture is reversed. Now most of the products show up in the channel $\mathrm{D}+\mathrm{OH}$ as shown in Fig. 10. We have calculated the norm of the wave packets in each channel. The results are given in Table II. For dissociation out of the $|0,4\rangle$ state $36 \%$ of the amplitude goes into the $\mathrm{H}+\mathrm{OD}$ channel. The overall selectivity between the two dissociation pathways is, accordingly, still quite modest. Note the small wave connecting the two channels in Fig. 10(b). This structure implies that the total breakup channel $\mathrm{H}+\mathrm{O}+\mathrm{D}$ can get some (small) population. In order to get a fully converged
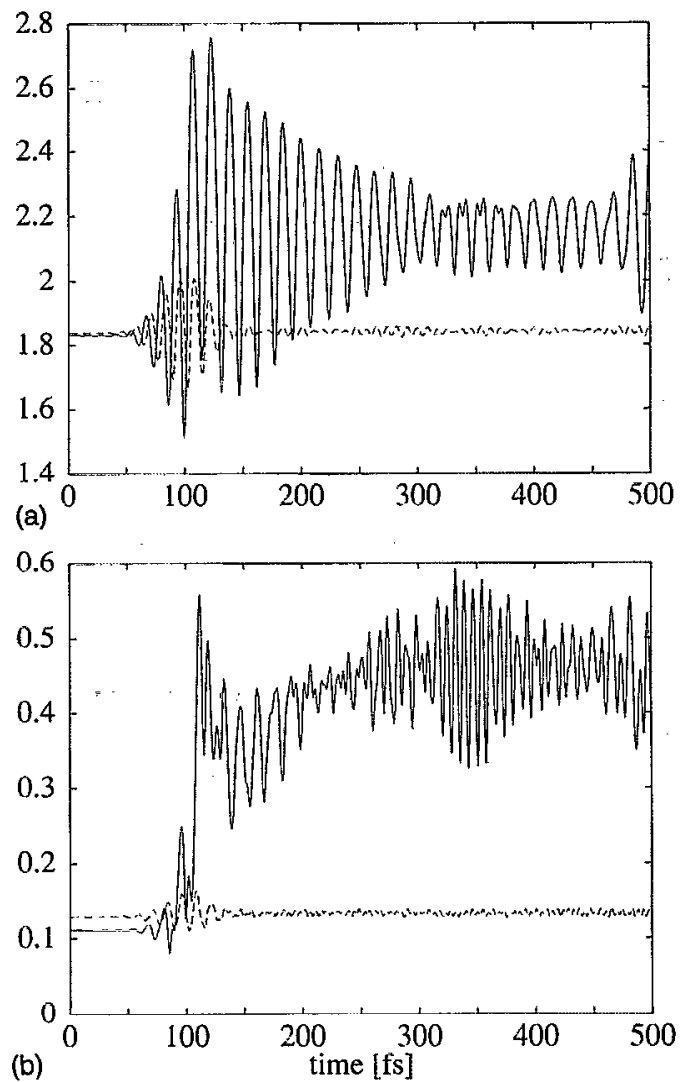

FIG. 5. Same as Fig. 4, except that $\omega_{\mathrm{ir}}=2599 \mathrm{~cm}^{-1}$, the full and dashed lines are associated with the $\mathrm{OD}$ bond and $\mathrm{OH}$ bond, respectively.

result for this channel a very large grid would be required (larger than the $256 \times 256$ grid used here).

These results for dissociation out of vibrational eigenstates using an ultrashort pulse are related to the results where a cw laser is used. ${ }^{24}$ Dissociation implies that freely moving fragments will show up,

$$
\exp \left(-i \hat{H}_{e} t / \hbar\right)|\phi(0)\rangle \stackrel{t \rightarrow \infty}{\rightarrow}\left|\phi_{\mathrm{OD}}(t)\right\rangle+\left|\phi_{\mathrm{OH}}(t)\right\rangle,
$$

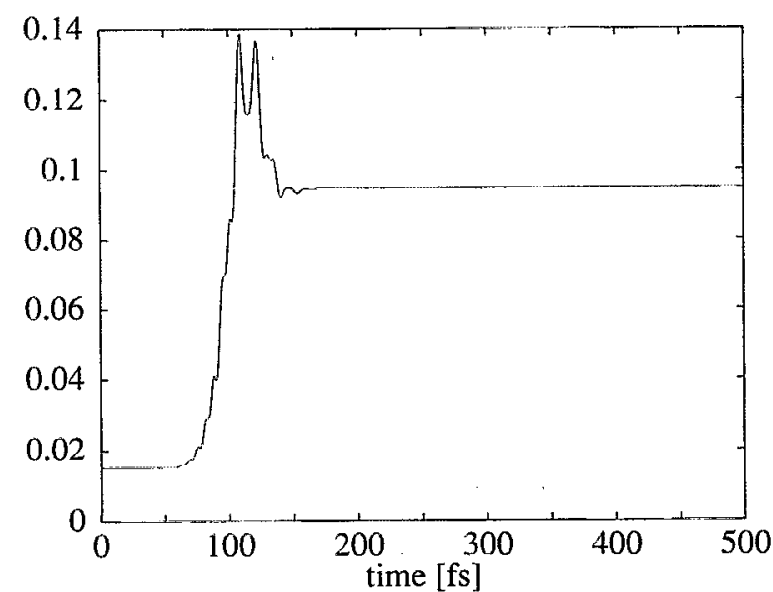

FIG. 6. Average energy of the HOD molecule under the same conditions as in Fig. 5. 


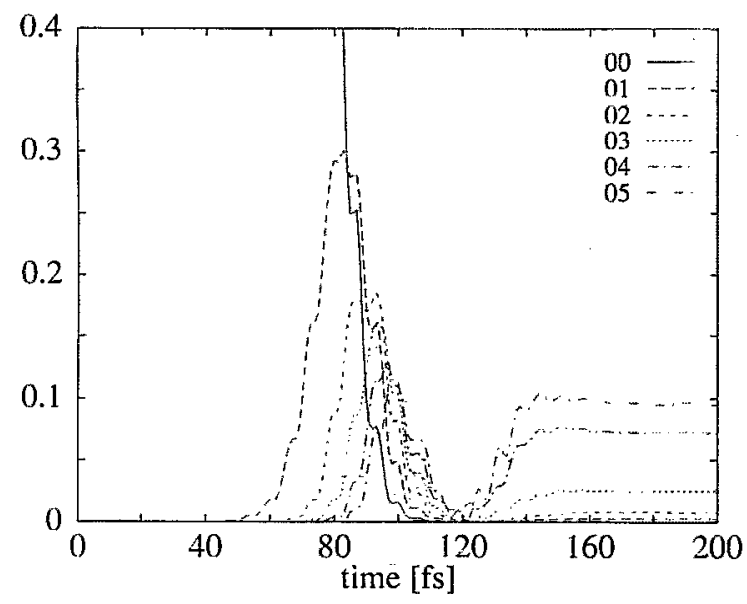

FIG. 7. Projections on vibrational eigenstates under the same conditions as in Fig. 5. where $\left|\phi_{\mathrm{OD}}(t)\right\rangle$ and $\left|\phi_{\mathrm{OH}}(t)\right\rangle$ are asymptotic wave packets (the atom-diatom potential has decayed to zero) for the $\mathrm{H}+\mathrm{OD}$ and the $\mathrm{D}+\mathrm{OH}$ channels, respectively, and

$$
|\phi(0)\rangle=\mathscr{C} \mu_{g e}\left|n_{\mathrm{OH}}, n_{\mathrm{OD}}\right\rangle
$$

where $\mathscr{C}$ is a constant. In the ultrashort pulse limit, the probability of observing products in, say, channel $\mathrm{H}+\mathrm{OD}$ is

$$
P_{\mathrm{OD}}^{\delta}=\left\langle\phi_{\mathrm{OD}}\left(t_{f}\right) \mid \phi_{\mathrm{OD}}\left(t_{f}\right)\right\rangle,
$$

where $t_{f}$ is the final propagation time and we used the fact that the wave functions associated with the two channels do not overlap. The corresponding result for a cw laser at frequency $\omega$ is

$$
P_{\mathrm{OD}}^{\mathrm{cW}}(\omega)=\int_{-\infty}^{\infty} d \tau e^{i \omega \tau}\left\langle\phi_{\mathrm{OD}}\left(t_{f}\right) \mid \phi_{\mathrm{OD}}\left(t_{f}+\tau\right)\right\rangle .
$$

We notice,

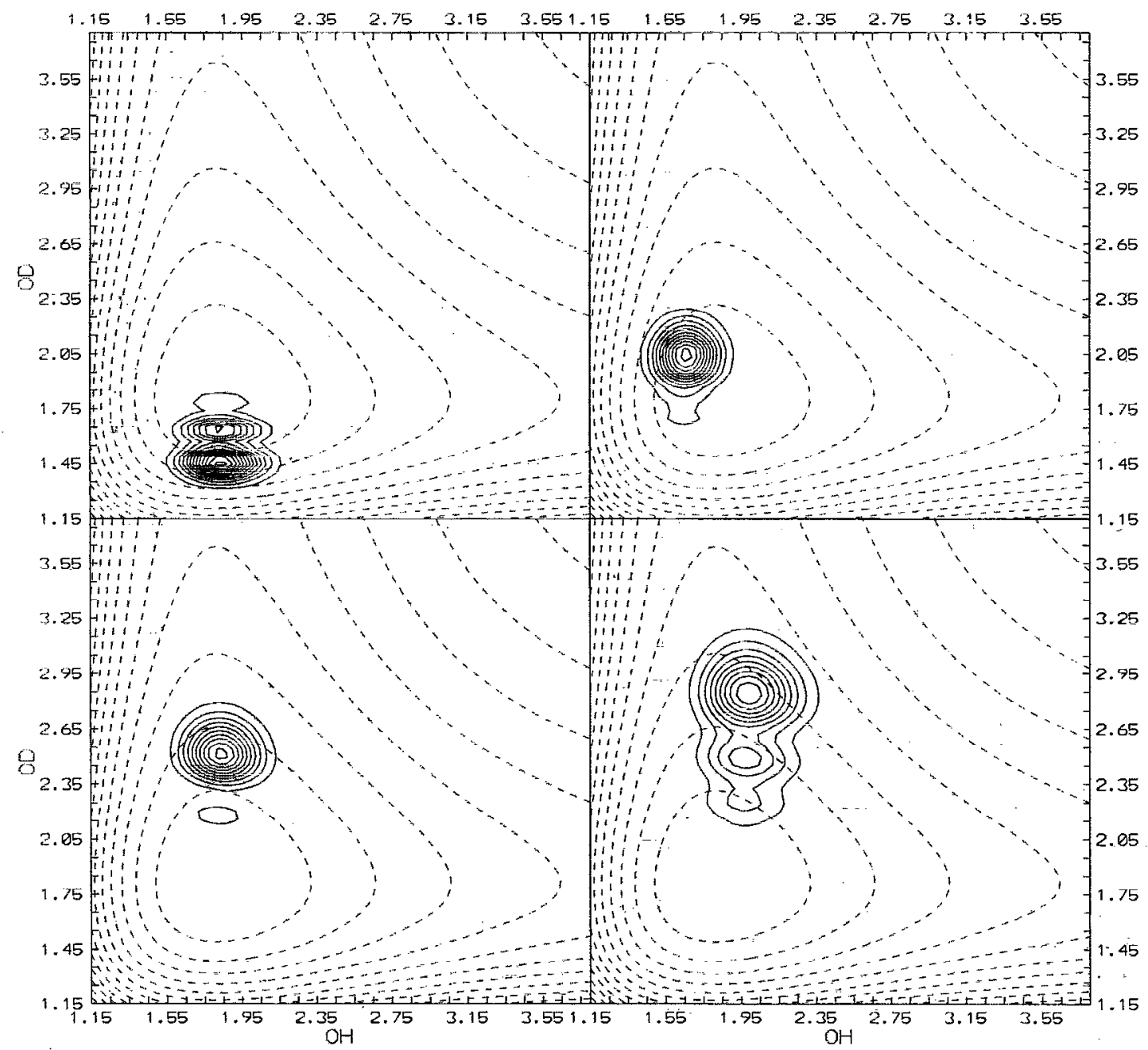

FIG. 8. Absolute square of the (nonstationary) state created with the Gaussian pulse of Fig. 5 superimposed on the potential energy surface of the electronic ground state (dashed lines). The wave packet is shown at $100 \mathrm{fs}$ (upper left panel), $102.5 \mathrm{fs}$ (upper right panel), $105 \mathrm{fs}$ (lower left panel), and 107.5 fs (lower right panel), respectively. 

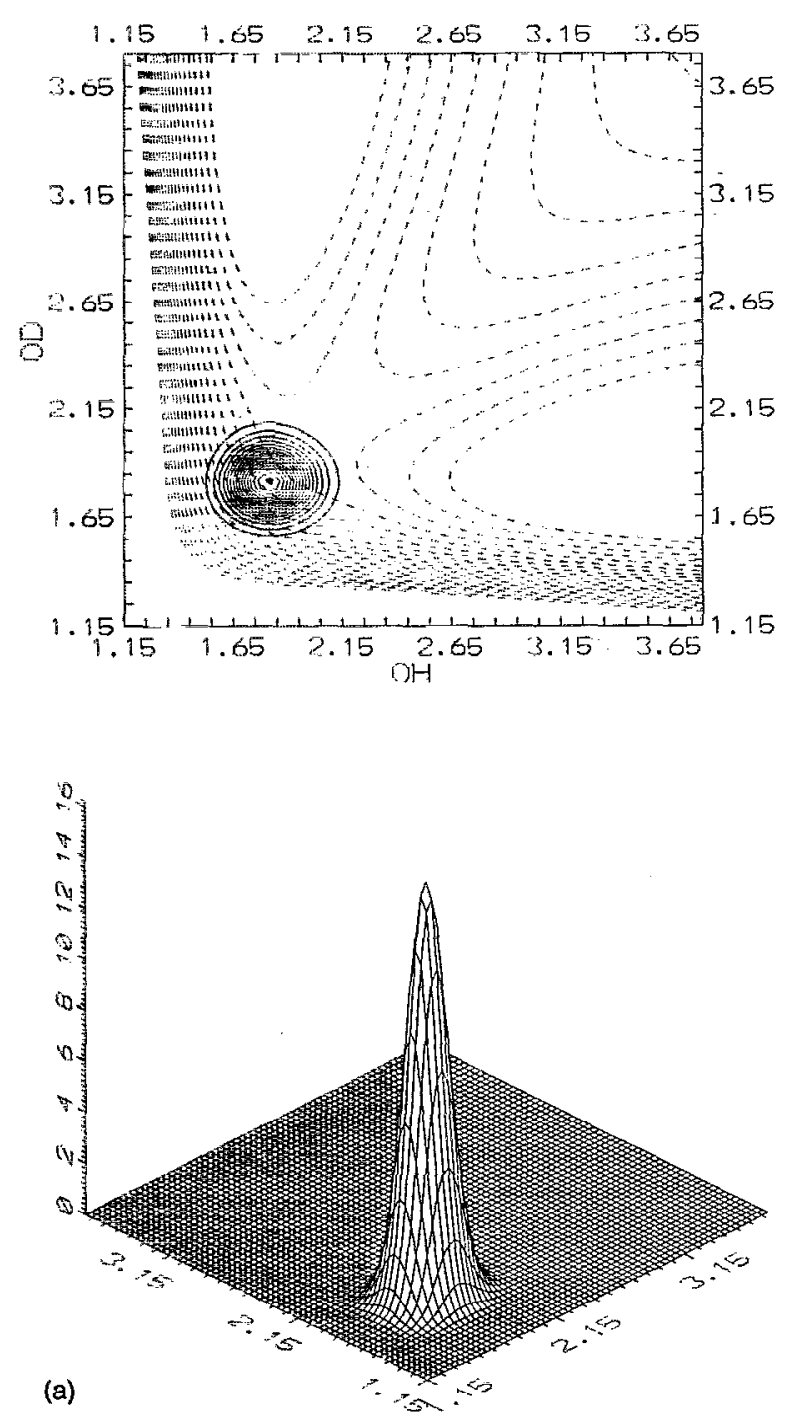

$$
\begin{aligned}
\int d \omega P_{\mathrm{OD}}^{\mathrm{W}}(\omega) & =2 \pi \int_{-\infty}^{\infty} d \tau \delta(\tau-0)\left\langle\phi_{\mathrm{OD}}\left(t_{f}\right) \mid \phi_{\mathrm{OD}}\left(t_{f}+\tau\right)\right\rangle \\
& =2 \pi P_{\mathrm{OD}}^{\delta}
\end{aligned}
$$

Since equivalent relations holds for the $\mathrm{D}+\mathrm{OH}$ channel, we get

$$
P_{\mathrm{OD}}^{\delta} / P_{\mathrm{OH}}^{\delta}=\frac{s d \omega P_{\mathrm{OD}}^{\mathrm{cw}}(\omega)}{\int d \omega P_{\mathrm{OH}}^{\mathrm{cw}}(\omega)} .
$$

Thus the branching ratio obtained when an ultrashort ( $\delta$ function) pulse is applied is equal to the overall branching ratio obtained when the absorption band is scanned with a $\mathrm{cw}$ laser.

Can we control the reaction pathway such that, say, only $\mathrm{D}+\mathrm{OH}$ is produced? In Fig. 11 the dissociation dynamics for the OD local mode (nonstationary) state created after $102.5 \mathrm{fs}$ is shown. We observe that, indeed, almost complete selectivity is obtained. The question arises whether other nonstationary local mode excitations of OD show the same kind of selectivity. The results are presented in Table II. The states $|100 \mathrm{fs}\rangle$ and $|105 \mathrm{fs}\rangle$ show a very
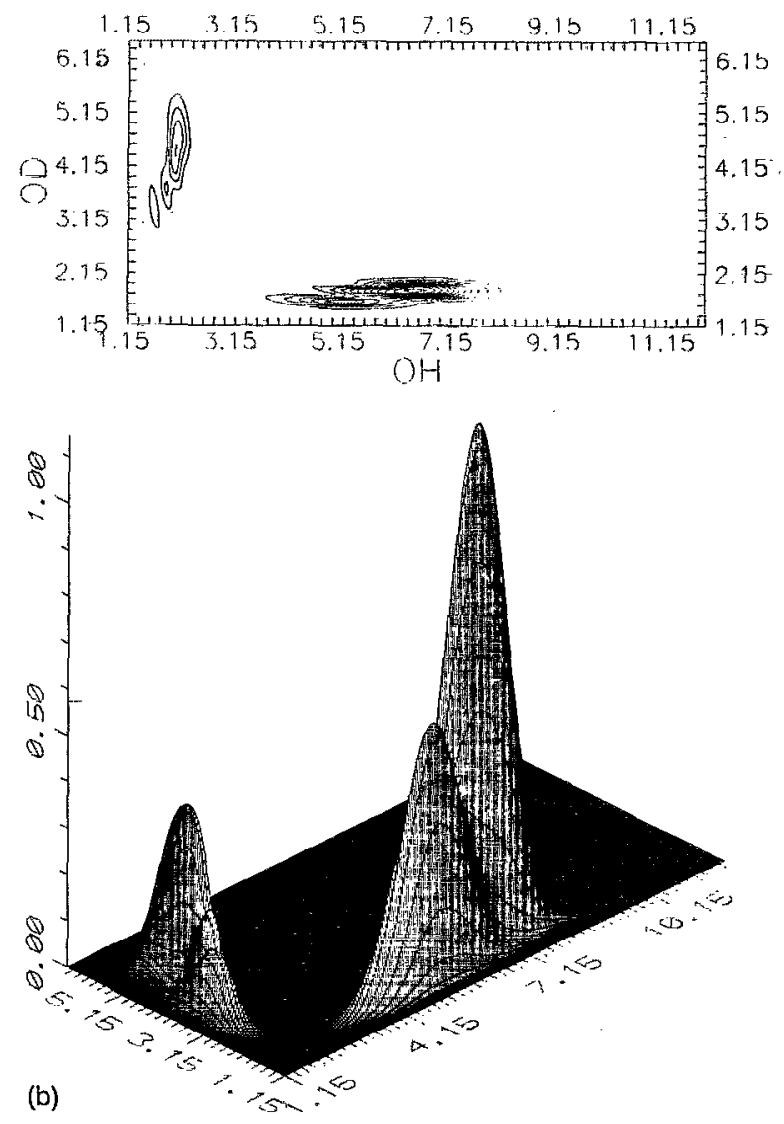

FIG. 9. (a) The absolute square of the product between the $|0,0\rangle$ state and the transition dipole function superimposed on the potential energy surface of the first electronically excited state (dashed lines). (b) Dissociation dynamics in the first electronically excited state of HOD starting with the state of (a). The absolute square of the wave function is shown after $15 \mathrm{fs}$ time evolution.

high selectivity, too. The fact that the state $|100 \mathrm{fs}\rangle$ dissociates, almost exclusively, into the $\mathrm{D}+\mathrm{OH}$ channel seems at first a little surprising. The average momentum in the direction of the OD bond in the electronic ground state is essentially zero since the bond is at the (inner) turning point. After excitation to the excited electronic state a high momentum is, however, created because the wave packet starts out on a very steep part of the potential energy surface.

The results in Table II map the selectivity with respect to the time delay to the second ultrashort ( $\delta$-function) pulse. A laser pulse with a finite pulse length creates a superposition of the packets created by a $\delta$-function pulse. From Table II one obtains, accordingly, an indication of the finite (short) pulse length which is required in order to obtain a high overall selectivity. This pulse length is of the order of $5 \mathrm{fs}$. We have tested the validity of this reasoning-a short Gaussian laser pulse (FWHM of $5 \mathrm{fs}$ ) centered at $t_{\mathrm{uv}}=103 \mathrm{fs}$ and with a (central) frequency, $\omega_{\mathrm{uv}}$, in the uv region was considered explicitly. The results are presented in Table III. These results demonstrate that, es- 

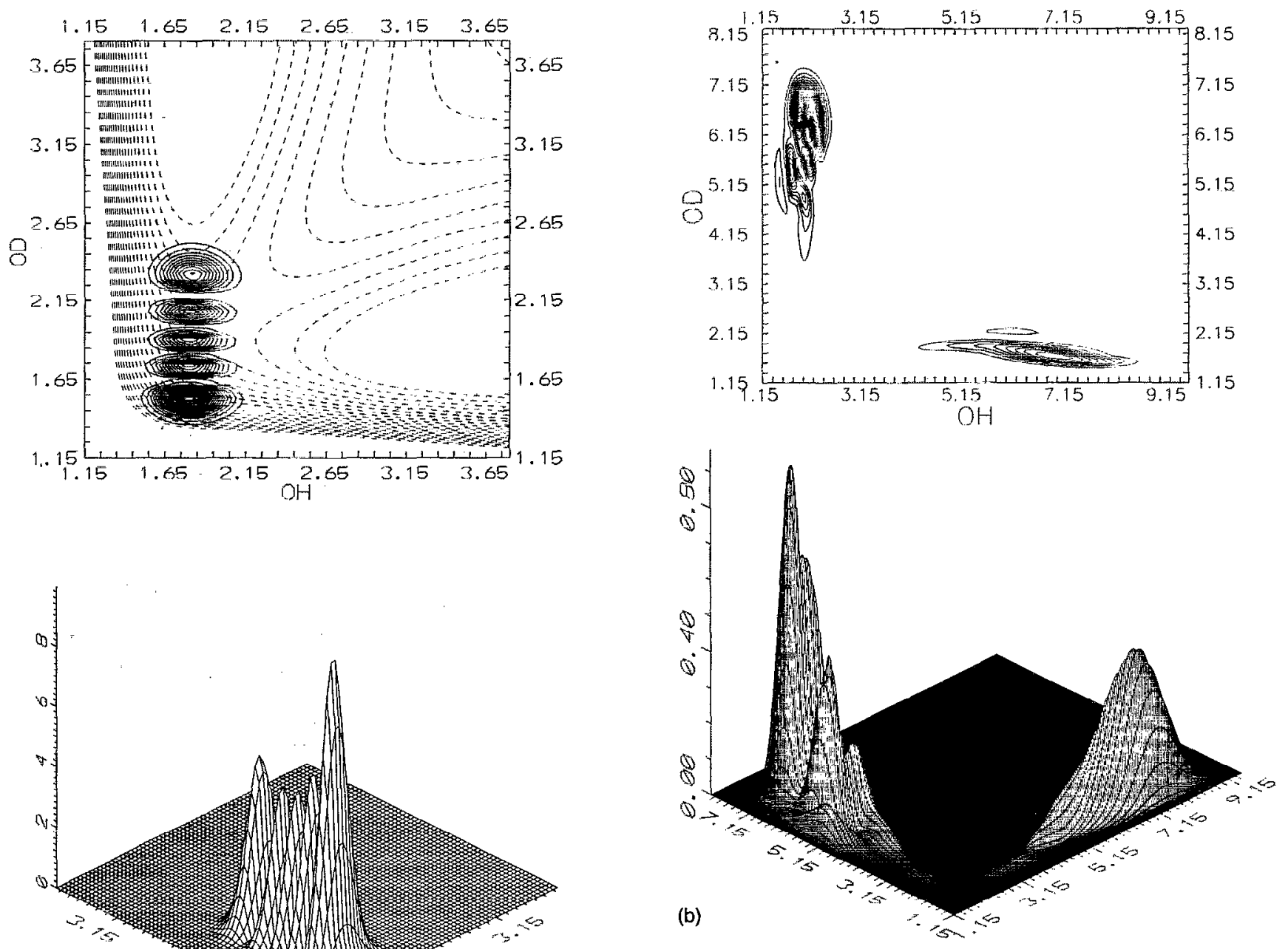

(a)

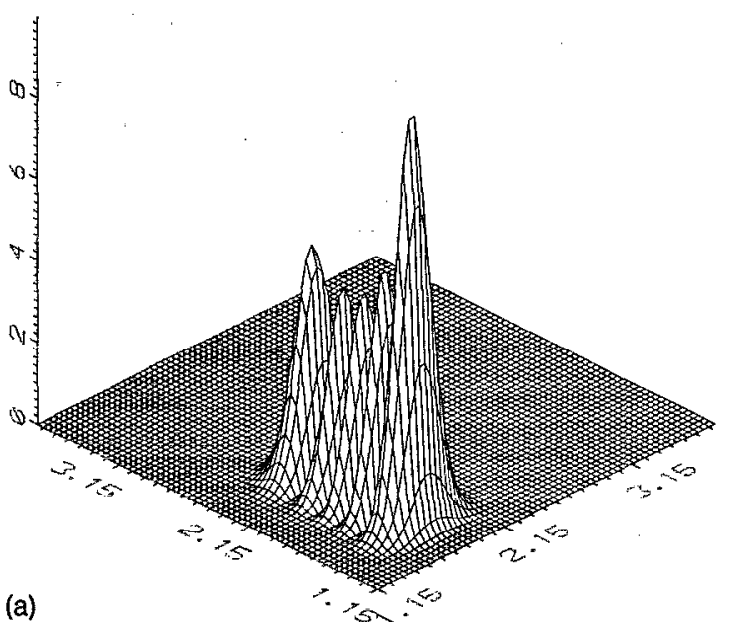

(b)

FIG. 10. (a) Same as Fig. 9(a), except that the initial state is $|0,4\rangle$, i.e., zero quanta in the OH stretch and four quanta in the OD stretch. (b) Same as Fig. $9(\mathrm{~b})$, except that the initial state is given in Fig. 10(a).

sentially, complete selectivity can be obtained over the entire uv absorption band. Figure 12 shows the dissociation dynamics at the frequency $\omega_{\mathrm{uv}}=54869 \mathrm{~cm}^{-1}$. The wave packet is as expected somewhat broadened compared to an excitation with a $\delta$-function pulse. The uncertainty in the

TABLE II. Total branching ratio between $\mathrm{D}+\mathrm{OH}$ and $\mathrm{H}+\mathrm{OD}$ in the first electronically excited state of HOD. The probability associated with these channels are given for various initial vibrational states of the electronic ground state. The nonstationary states, $|x . x \hat{\mathrm{f}}\rangle$, are electronically excited by a $\delta$-function pulse.

\begin{tabular}{lcc}
\hline Initial state & D+OH & H+OD \\
\hline$|0,0\rangle$ & 0.24 & 0.76 \\
$|0,4\rangle$ & 0.64 & 0.36 \\
$|100.0 \mathrm{fs}\rangle$ & 0.98 & 0.02 \\
$|102.5 \mathrm{fs}\rangle$ & 0.98 & 0.02 \\
$|105.0 \mathrm{fs}\rangle$ & 0.96 & 0.04 \\
$|107.5 \mathrm{fs}\rangle$ & 0.71 & 0.29 \\
\hline
\end{tabular}

frequency for such a short pulse is substantial, the full width at half-maximum (FWHM) in the frequency domain is $5888 \mathrm{~cm}^{-1}$, i.e., it is about $10 \%$ of the central frequency. Concerning the results in Table III, it should be noticed that above $\sim 70000 \mathrm{~cm}^{-1}$ the total breakup channel, $\mathrm{H}+\mathrm{O}+\mathrm{D}$, plays a role. We did not analyze the population in this channel separately, that would require a large computation (with a grid size larger than $256 \times 256$ ) and, in addition, the aim of the present study was to control the competition between the channels $\mathrm{H}+\mathrm{OD}$ and $\mathrm{D}+\mathrm{OH}$ in the energy regime where only these channels are open. The numbers in parenthesis are not fully converged-all products where the $\mathrm{O}-\mathrm{H}$ distance is larger than the $\mathrm{O}-\mathrm{D}$ distance were classified as $\mathrm{H}+\mathrm{OD}$ and an equivalent criterion for the $\mathrm{D}+\mathrm{OH}$ channel was used throughout this study.

Duc to the fast vibrational dynamics of the electronic ground state, much of the selectivity in the fragmentation pathway is lost if the dissociation is initiated with a pulse of 


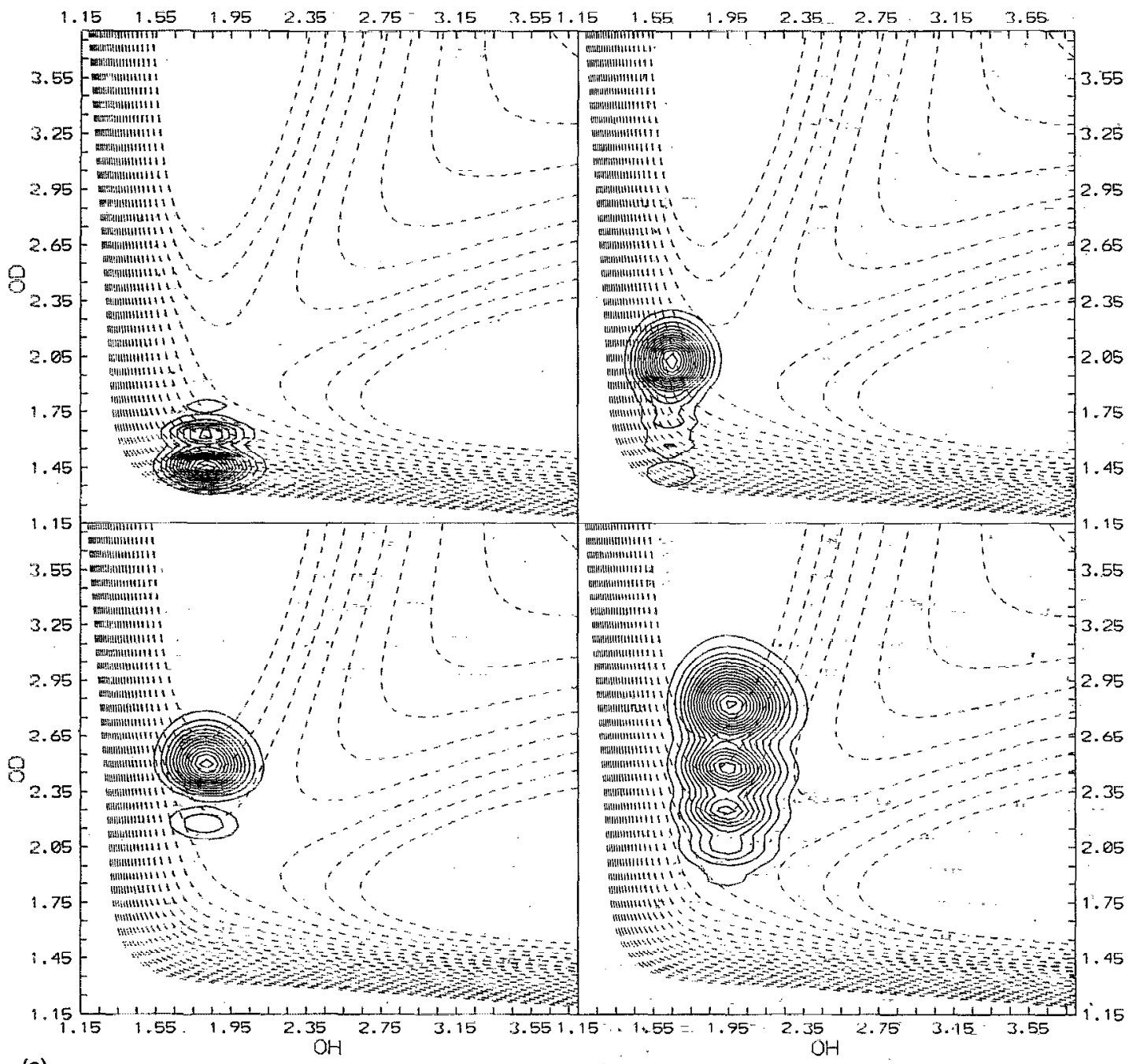

(a)
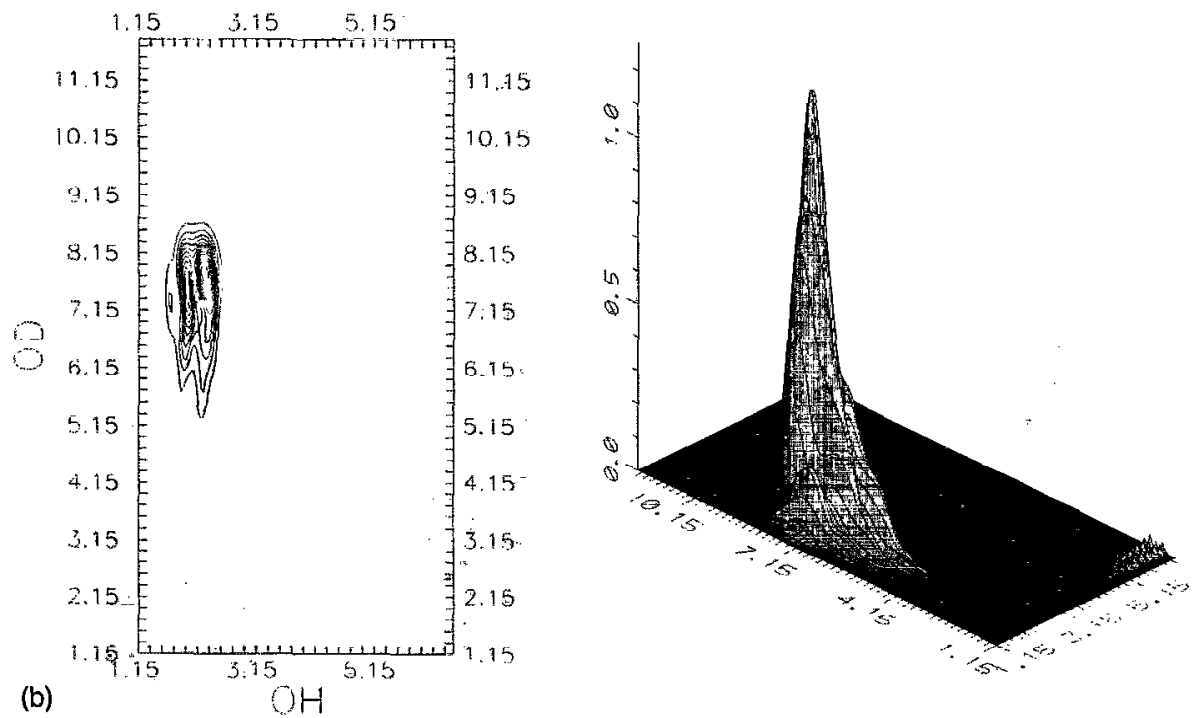

FIG. 11. (a) The (nonstationary) states of Fig. 8 multiplied by the square of the transition dipole function. The wave functions are superimposed on the potential energy surface of the first electronically excited state (dashed lines). (b) Dissociation dynamics in the first electronically excited state of HOD starting with the (nonstationary) state created after $102.5 \mathrm{fs}$ time evolution in the electronic ground state [(a), upper right corner]. The figure shows the absolute square of this function after $15 \mathrm{fs}$ time evolution in the dissociative state. 
TABLE III. Total electronic transition probability $\left(P_{\text {tot }}\right)$ and branching ratio between $\mathrm{D}+\mathrm{OH}$ and $\mathrm{H}+\mathrm{OD}$ in the first electronically excited state of HOD. The excitation is carried out with a Gaussian pulse on a molecule under the ir excitation of Fig. 5. The duration of the pulse is $5 \mathrm{fs}$ (FWHM), it is centered at $t_{\mathrm{uv}}=103 \mathrm{fs}$, and the frequency, $\omega_{\mathrm{uv}}$, is specified in the table. Numbers in parenthesis are not fully converged due to the presence of the $\mathrm{H}+\mathrm{O}+\mathrm{D}$ channel, see the text.

\begin{tabular}{cccc}
\hline \hline$\omega_{\mathrm{uv}}\left(\mathrm{cm}^{-1}\right)$ & $P_{\text {tot }}$ & $\mathrm{D}+\mathrm{OH}$ & $\mathrm{H}+\mathrm{OD}$ \\
\hline 32921 & 0.004 & 1.00 & 0.00 \\
43895 & 0.027 & 1.00 & 0.00 \\
54869 & 0.100 & 0.98 & 0.02 \\
65842 & 0.371 & 0.98 & 0.02 \\
76816 & 0.039 & $(0.94)$ & $(0.06)$ \\
87790 & 0.001 & $(0.63)$ & $(0.37)$ \\
\hline
\end{tabular}

much longer duration than $\sim 5 \mathrm{fs}$. Thus a $10 \mathrm{fs}$ pulse $\left(t_{\mathrm{uv}}\right.$ $=103 \mathrm{fs}, \omega_{\mathrm{uv}}=54869 \mathrm{~cm}^{-1}$ ) gives $83 \%$ in the $\mathrm{D}+\mathrm{OH}$ channel and $17 \%$ in the $\mathrm{H}+\mathrm{OD}$ channel. The exact timing of the second pulse is, indeed, very critical.

Finally, we have in the calculations reported above assumed that the phase difference between the two fields is zero-a phase difference cannot affect the branching ratio in the scheme considered in the present work. Even if this proposition was wrong, any possible effect of a phase difference will tend to average out since the frequencies of the two lasers are highly incommensurate (differs by a factor of more than 10). Furthermore, in order to test this claim, we have repeated the calculation for a 5 fs pulse at $\omega_{\mathrm{uv}}$ $=65842 \mathrm{~cm}^{-1}$ (Table III) after introducing a phase shift in the pulse, i.e., instead of the simple cosine function, we use $\cos \left(\omega_{\mathrm{uv}} t+\delta\right)$. The results for $\delta=3.0$ and $\delta=5.0$, respectively, are to more than four significant figures identi- cal to the result at zero phase difference. Thus a phase difference between the two laser fields plays a negligible role for our results.

\section{SUMMARY}

We have shown that complete control of the fragmentation pathway in the photodissociation of HOD can be obtained. The method suggested here involves the dissociation of a nonstationary local mode vibrational state of HOD. Such a state is created using an intense laser in the ir region. A second ultrashort laser pulse dissociates the molecule via an excitation to an excited electronic state. This laser is fired when the vibrating molecule has a convenient position and/or momentum distribution. Compared to the well-established setup where dissociation out of a stationary local mode eigenstate of HOD is considered, the method suggested here is superior-essentially due to the fact that vibrational eigenstates always have some amplitude in the Franck-Condon region which-in an uncontrollable way-can evolve into either of the two product channels. This advantage is, obviously, gained at the expense of experimental conditions in terms of laser powers, timing, and pulse durations, which are at the limits of present technology. Thus ultrashort ( $\sim 5 \mathrm{fs}$ ) laser pulses in the uv are not available at present. The required time delay with femtosecond accuracy is, however, already experimentally obtainable since the needed micrometer accuracy in the path length can be handled. Our study has, nevertheless, shown what can be achieved by an active control scheme in the time domain.

The calculations were based on a well-established two degrees of freedom model of water using two high quality
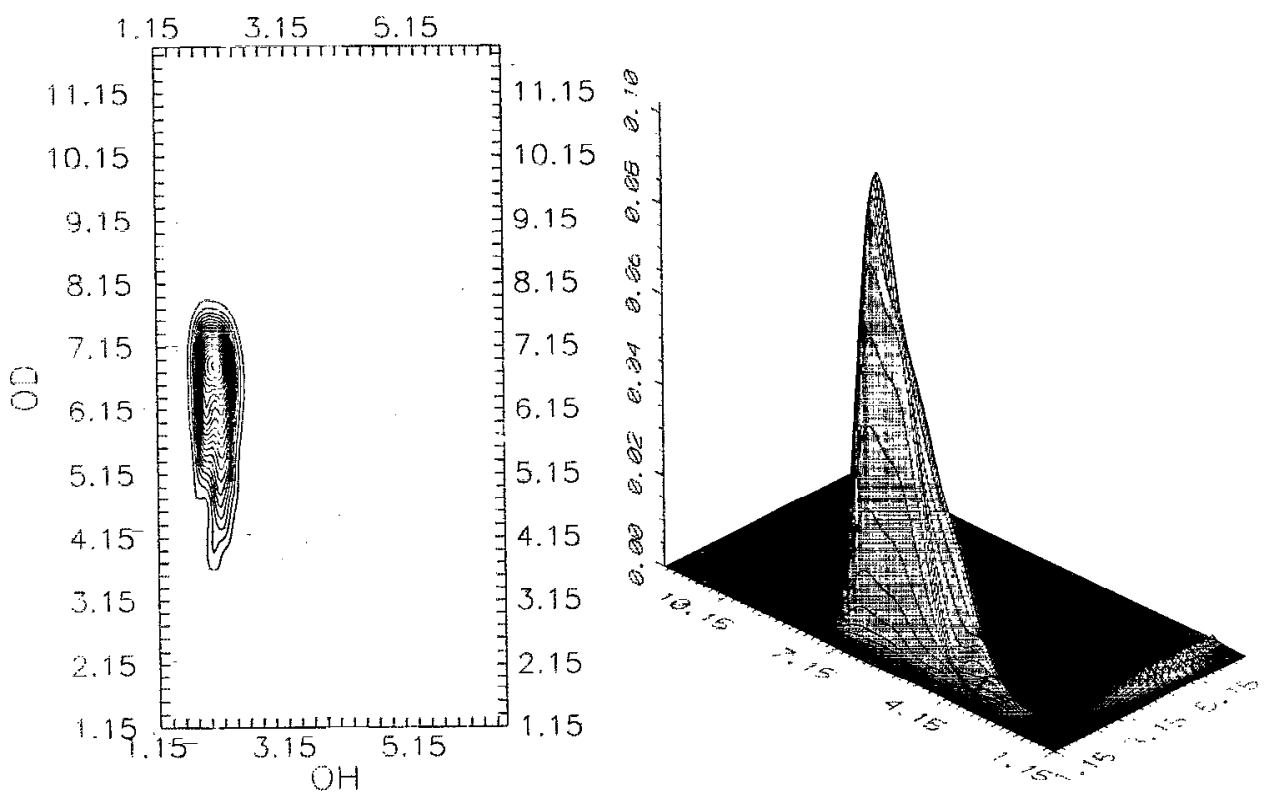

FIG. 12. Dissociation dynamics in the first electronically excited state of HOD induced by a 5 fs (FWHM) Gaussian laser pulse. The pulse is centered at $t_{\mathrm{uv}}=103 \mathrm{fs}$ and the (central) frequency is $54869 \mathrm{~cm}^{-1}$. The vibrational state of the electronic ground state is nonstationary as described in Fig. 5 . The total propagation time is $117.5 \mathrm{fs}$. 
potential energy surfaces and (numerically) exact quantum solutions to the nuclear dynamics. The electronic dipole surfaces used in the coupling terms to the laser fields are, obviously, not highly accurate but simple model functions. We do not expect that the use of more accurate dipole surfaces will change the basic features of the results reported here. We did, in fact, test the importance of the transition dipole surface on the branching ratio. We found branching ratios which deviates from the results of Table II only by a few percent when the transition dipole was taken to be a constant.

When considering the application of the control scheme studied in this paper to other photodissociation processes, it should be noted that the HOD molecule in many ways is an ideal system. On the other hand, the strong anharmonicity and the light masses of the molecule make the timing of the second laser quite critical. Heavier masses and more harmonic potentials would give slower vibrations and more coherent wave packets. That would be easier to handle. Furthermore, bond-selective excitations of the type studied here are not restricted to simple molecules, which contains local mode eigenstates. It has recently been demonstrated that bond-selective excitations in molecules without local mode eigenstates can be created via intense laser pulses with optimized shapes. ${ }^{25}$

\section{ACKNOWLEDGMENT}

This research was supported by the Danish Natural Science Research Council.
${ }^{1}$ F. F. Crim, Science 249, 1387 (1990).

${ }^{2}$ V. Engel and R. Schinke, J. Chem. Phys. 88, 6831 (1988).

${ }^{3}$ D. G. Imre and J. Zhang, Chem. Phys. 139, 89 (1989).

${ }^{4}$ R. L. Vander Wal, J. L. Scott, and F. F. Crim, J. Chem. Phys. 92, 803 (1990).

${ }^{5}$ I. Bar, Y. Cohen, D. David, S. Rosenwaks, and J. J. Valentini, J. Chem. Phys. 93, 2146 (1990).

${ }^{6}$ R. L. Vander Wal, J. L. Scott, F. F. Crim, K. Weide, and R. Schinke, J. Chem. Phys. 94, 3548 (1991).

${ }^{7}$ W. Jakubetz, J. Manz, and V. Mohan, J. Chem. Phys. 90, 3686 (1989).

${ }^{8}$ W. Jakubetz, B. Just, J. Manz, and H.-J. Schreier, J. Phys. Chem. 94, 2294 (1990).

${ }^{9}$ S. Chelkowski, A. D. Bandrauk, and P. B. Corkum, Phys. Rev. Lett. 65 , 2355 (1990).

${ }^{10}$ S. A. Rice, D. J. Tannor, and R. Kosloff, J. Chem. Soc., Faraday Trans. 2 82, 2423 (1986).

${ }^{11}$ D. J. Tannor, R. Kosloff, and S. A. Rice, J. Chem. Phys. 85, 5805 (1986).

${ }^{12}$ P. Brumer and M. Shapiro, Chem. Phys. Lett. 126, 541 (1986).

${ }^{13}$ P. Brumer and M. Shapiro, Chem. Phys. 139, 221 (1989).

${ }^{14}$ D. Kosloff and R. Kosloff, J. Comp. Phys. 52, 35 (1983).

${ }^{15}$ C. Leforestier, R. Bisseling, C. Cerjan, M. D. Feit, and R. Friesner, J. Comp. Phys. 94, 59 (1991)

${ }^{16}$ R. Kosloff and H. Tal-Ezer, Chem. Phys. Lett. 127, 223 (1986).

${ }^{17}$ J. R. Reimers and R. O. Watts, Mol. Phys. 52, 357 (1984).

${ }^{18}$ J. Zhang, D. G. Imre, and J. H. Frederick, J. Phys. Chem. 93, 1840 (1989).

${ }^{19}$ R. T. Lawton and M. S. Child, Mol. Phys. 40, 773 (1980).

${ }^{20}$ B. J. Rosenberg, W. C. Ermler, and I. Shavitt, J. Chem. Phys. 65, 4072 (1976).

${ }^{21}$ V. Staemmler and A. Palma, Chem. Phys. 93, 63 (1985).

${ }^{22}$ V. Engel, R. Schinke, and V. Staemmler, J. Chem. Phys. 88, 129 (1988).

${ }^{23}$ R. B. Walker and R. K. Preston, J. Chem. Phys. 67, 2017 (1977).

${ }^{24}$ N. E. Henriksen, Chem. Phys. Lett. 169, 229 (1990).

${ }^{25}$ S. Shi and H. Rabitz, Chem. Phys. 139, 185 (1989). 